\title{
Einführung zum Vortrag von Horst Eberhard Richter
}

\author{
Klaus Antons
}

Im Jahre 1974 erschien ein Buch, das für Aufsehen sorgte: „Lernziel Solidarität“ von Horst-Eberhard Richter (Richter 1974). Es war keineswegs seine erste Veröffentlichung - allerdings waren seine wichtigen früheren Bücher eher etwas für Fachleute.

1963 erschien „Eltern, Kind und Neurose“; zusammen mit dem später veröffentlichten „Patient Familie“ (Richter 1963, 1970) gehörte es zu den ersten Werken über Familiendynamik und Familientherapie im deutschsprachigen Raum. Beide Arbeiten brachten den Diskurs über Familiendynamik maßgeblich in Schwung.

1972 kam „Die Gruppe - Hoffnung auf einen neuen Weg, sich selbst und andere zu befreien" heraus: ein Buch mit einem längstmöglichen Untertitel, das Richter über den engeren Kreis von Fachleuten hinaus bekannt machte und für die Generation jüngerer Gruppendynamiker und Gruppendynamikerinnen, die sich seit einem halben Jahrzehnt mit Gruppen abmühten, ein Hoffnungsstrahl war (Richter 1972). Heute sind wir alle etwas realistischer geworden.

War schon in „Die Gruppe“ das politische Moment angesprochen, so wird zwei Jahre später, mit „Lernziel Solidarität“", Horst Eberhard Richter ganz zu dem, was er seitdem geblieben ist: ein durch und durch politisch denkender und handelnder Mensch. Nicht zu Unrecht wurde er vor einigen Jahren als der „Analytiker der Nation“ bezeichnet: „Wie kein zweiter Psychotherapeut reagiert Richter auf die sozialen und politischen Themen seiner Zeit - ein unermüdlicher < Analytiker und Therapeut seines Landes >“, wie Johannes Rau es formulierte.

Um welche Solidaritäten ging es 1974? Zunächst um die zwischen den Geschlechtern, um die „Notwendigkeit, sich miteinander zu emanzipieren“; um die Entdeckung von Solidarität in Selbsthilfegruppen; um ,Versuche gemeinsamer Umerziehung in spontanen Gruppen"; und schließlich um die Solidarisierung nach unten - mit Randgruppen unserer Gesellschaft. Manche der damals gestellten Postulate sind heute eingelöst, manche stellen sich heute nicht mehr oder sehr anders. Beim jetzigen Lesen des Textes wird deutlich, wie vieles davon auch heute noch brandaktuell ist. Richter hat bereits damals auf die tief

Online publiziert: 03.01 .2013

(C) Springer Fachmedien Wiesbaden 2012

Dr. phil. habil. Dipl. Psych. K. Antons $(\bowtie)$

Büsingen, Deutschland

E-Mail: antons.buesingen@t-online.de 
verwurzelte Denktradition des Individuums hingewiesen, die viele Versuche zunichtemacht, Kommunikation und Solidarität in Gruppen zu verwirklichen.

Er hat viele weitere Veröffentlichungen auf seiner Liste, von denen „Der Gotteskomplex" die vielleicht wichtigste ist. Immer hat er den Leser und die Leserin an seinem intellektuellen und menschlichen Entwicklungsweg teilhaben lassen. Auch Vorträge über den „Abschied von der Egomanie“ (Lindauer Psychotherapiewochen 2002) oder das, was er dem österreichischen Schwesterverband des DAGG zu seinem runden Jubiläum ins Stammbuch geschrieben hat (Richter 2009), zeigen: Nicht nur die Analyse - mit unerbittlicher Schärfe - sondern auch das unermüdliche Plädoyer für eine Kultur der Verständigung waren ihm ein Herzensanliegen.

Er hat nicht nur geschrieben, sondern auch gehandelt. $\mathrm{Ob}$ es die Friedensbewegung war, die Initiativgruppe Eulenkopf, Ärzte gegen den Atomkrieg, oder bis zum Schluss attac: Er war jemand, der sich mit Leidenschaft für etwas engagierte, was ihm wichtig war.

Wer war Horst Eberhard (beide Vornamen waren ihm wichtig) Richter? Er wurde 1923 in Berlin geboren, wurde Hitlerjunge, war im Arbeitsdienst und wurde mit 18 Soldat - seine Kriegserfahrungen in Russland sowie die Ermordung seiner Eltern kurz nach Kriegsende haben sein lebenslanges Friedensengagement stark geprägt.

Er promovierte im Medizin und Psychologie. Seine berufliche Karriere führte ihn von 1959 bis 1962 als ärztlichen Leiter der „Beratungs- und Forschungsstelle für seelisch gestörte Kinder und Jugendliche“ nach Berlin-Wedding und von 1962 bis 1992 als Direktor des „Zentrums für Psychosomatische Medizin“ und Prof. an der Universität nach Gießen. 1982 begründete er die deutsche Sektion der „Internationalen Ärzte zur Verhütung des Atomkrieges und für soziale Verantwortung“" (IPPNW) mit und war dort bis zu seinem Tod im Jahre 2011 Ehrenvorstand. 1988 bis 1992 engagierte er sich im Board of Directors der ,International Foundation for the Survival and the Development of Humanity“ (Moskau), schliesslich wirkte er von 1992 bis 2002 als geschäftsführender Direktor des Sigmund Freud-Instituts, Frankfurt am Main.

Für diese umfassende Tätigkeit erhielt er unter anderem 1980 den Theodor-HeussPreis, 2001 den Fairness-Ehrenpreis, 2002 die Goethe-Plakette der Stadt Frankfurt und 2008 die Paracelsus-Medaille der Deutschen Ärzteschaft.

Für die Veranstalter der Berliner Fachtagung „Kann Solidarität unsere Zukunft retten?" war es eine Ehre und große Freude, dass Horst-Eberhard Richter trotz seiner damals 87 Lebensjahre zum Eröffnungsvortrag zusagte und einem Titelvorschlag eine markante Wendung gab, indem er auf die Frage eine Antwort formulierte: „Ohne Solidarität keine Zukunft!“. Er liess in seinem Vortrag, der sich bisweilen vom geschriebenen Text löste, die Zuhörenden in sehr persönlicher Weise an seinem Lebensweg und seinem Bemühen um Verstehen und Versöhnung - und Solidarität als einer unabdinglichen Notwendigkeit - teilhaben. Es ist unvergesslich, wie dieser zart und gebrechlich wirkende Mann in voller Wachheit und geistiger Schärfe sprach - und sich nicht scheute, an einer sehr bewegenden Stelle alle an seiner Bewegtheit teilnehmen zu lassen. Darin zeigte er persönliche Grösse.

Babette Brinkmann (2010) schreibt über diesen Auftritt:

„Unter anderem fesselt er seine Zuhörer mit Einblicken in einen über Jahre dauernden Gesprächskreis mit Personen aus dem politischen und kulturellen Leben über alle Parteien und Bundesländer hinweg. Diese Gespräche wurden nicht ausgewertet oder ver- 
öffentlicht und dienten ausschliesslich dem Zweck, voneinander zu lernen, gegenseitiges Verständnis und neue Perspektiven zu entwickeln oder Handlungsoptionen zu erweitern. Die Faszination seines Vortrags mag neben der konsequenten Werteorientierung in Richters beruflichem Handeln in der gesellschaftlich verankerten Gruppenarbeit liegen. Es klingt nach gruppendynamischem Laboratorium ohne Labor".

Über eben diesen Gesprächskreis schreibt Gunter Hofmann in der ZEIT (Nr. 52 v. 22. 12. 2011, S. 48):

„Von Christa Wolf und Christoph Hein oder Friedrich Schorlemmer bis zu Marion Dönhoff, Richard von Weizsäcker, Antje Vollmer oder (gelegentlich) Oskar Lafontaine reichte die Bandbreite. Schwierigste Themen kamen zur Sprache, seelische Verwundungen, aber auch tiefe politische Diskrepanzen. Vorstellen muss man sich dann einen Moderator im Kreis, aber als exaktes Gegenbild zum Talkmaster. Alles setzte Richter dem Gespräch aus, alles war < Analyse >, ständig, mit leiser und sehr klarer Stimme geschah sie, und wie er das machte, steckte immer ein Stück Anerkennung für denjenigen darin, der sich äusserte“.

Zwischen Richters Auftritt auf der Solidaritäts-Tagung und der Fertigstellung dieses Manuskriptes liegen mehr als zwei Jahre. Das Erscheinen des Themenheftes kann er nicht mehr miterleben, leider können wir ihm dieses nicht mehr überreichen: Horst Eberhard Richter starb, im Alter von 87 Jahren, am 19. 12. 2011 in Giessen. Kurz zuvor hatte er, ebenfalls in Berlin, sein letztes Buch vorgestellt: „Moral in den Zeiten der Krise“. Sein Lebensthema, wie Hofmann in der ZEIT(a.a. O.) meint.

In der Presse wurde er posthum als „Befreier“ (ZEIT, a.a.O.) und als „Herold der Mündigkeit" (Steinfeld 2011) geehrt.

\section{Literatur}

Brinkmann, B. (2010). Bericht über die öffentliche Fachtagung der Sektion Gruppendynamik im DAGG/DGGO 2010 in Berlin. matrix 1/2010, S. 29.

Hofmann, G. (2011). Ein Befreier. DIE ZEIT Nr. 52 v. 22. 12. 2011, S. 48.

Richter, H. E. (1963). Eltern, Kind, Neurose. Reinbek: Rowohlt.

Richter, H. E. (1970). Patient Familie. Reinbek: Rowohlt.

Richter, H. E. (1972). Die Gruppe. Reinbek: Rowohlt.

Richter, H. E. (1974). Lernziel Solidarität. Reinbek: Rowohlt.

Richter, H. E. (2009). Die Gruppe als Lebens- und Therapiemodell. matrix 2/2009, S. 29-35.

Steinfeld, T. (2011). Der Herold der Mündigkeit. Süddeutsche Zeitung v. 21. 12. 2011, S. 11. 